\title{
BMJ Open Cardiovascular fitness and risk of migraine: a large, prospective population-based study of Swedish young adult men
}

\author{
Jenny Nyberg, ${ }^{01,2}$ Sara Gustavsson, ${ }^{3}$ Mattias Linde, ${ }_{7}^{4}$ N David Åberg, ${ }^{5,6}$ \\ Jessica L Rohmann, ${ }^{7}$ Maria Åberg, ${ }^{8,9}$ Tobias Kurth, ${ }^{\circ 7}$ Margda Waern, ${ }^{10,11}$ \\ Georg Hans Kuhn ${ }^{1,12}$
}

To cite: Nyberg J, Gustavsson S Linde M, et al. Cardiovascular fitness and risk of migraine: a large, prospective populationbased study of Swedish young adult men. BMJ Open 2019;9:e029147. doi:10.1136/ bmjopen-2019-029147

- Prepublication history for this paper is available online. To view these files, please visit the journal online (http://dx.doi. org/10.1136/bmjopen-2019029147).

Received 14 January 2019

Revised 04 July 2019

Accepted 23 July 2019
Check for updates

(C) Author(s) (or their employer(s)) 2019. Re-use permitted under CC BY-NC. No commercial re-use. See rights and permissions. Published by BMJ.

For numbered affiliations see end of article.

Correspondence to Dr Jenny Nyberg; jenny.nyberg@neuro.gu.se

\section{ABSTRACT}

Objectives To examine the longitudinal relationship between cardiovascular fitness in young adult men and future risk of migraine and to estimate eventual differential effects among categories of body mass index (BMI) and blood pressure.

Design National, prospective, population-based cohort study.

Setting Sweden 1968-2014.

Participants 18-year-old Swedish men ( $\mathrm{n}=1819$ 828) who underwent mandatory military conscription examinations during the years 1968-2005.

Primary and secondary outcomes The primary outcome was the first dispensation of prescribed migraine-specific medication, identified using the Swedish Prescribed Drug Register. The secondary outcome was documented migraine diagnosis from the Swedish National Hospital Register.

Results During follow-up, 22533 men filled a prescription for migraine-specific medication. After confounding adjustment, compared with high cardiovascular fitness, low and medium fitness increased the risk of migrainespecific medication (risk ratio $(\mathrm{RR})_{\text {low: }}: 1.29,95 \% \mathrm{Cl} 1.24$ to 1.35; population attributable fraction: $3.6 \%, 95 \% \mathrm{Cl} 1.7 \%$ to $5.3 \%$ and $\mathrm{RR}_{\text {medium }}: 1.15,95 \% \mathrm{Cl} 1.12$ to 1.19 ; population attributable fraction: $8.0 \%, 95 \% \mathrm{Cl} 4.0 \%$ to $11.7 \%$ ). To assess potential effect measure modification, stratified analyses of these association by levels of BMI and blood pressure showed that lower fitness levels increased risk of migraine across all groups except among underweight men or men with high diastolic blood pressure.

Conclusions Young men with a lower cardiovascular fitness had a higher long-term risk of developing pharmacological prescription-requiring migraine. This study contributes with information regarding risk factors for migraine in men, an understudied population in migraine research.

\section{INTRODUCTION}

Migraine is an intermittent neurological disorder with strong influences on the vascular system. ${ }^{1}$ Cardiovascular fitness, a state indicating overall capacity of the cardiovascular and respiratory systems and the ability to carry
Strengths and limitations of this study

- This is a large, longitudinal study of a nearly total population sample of young Swedish men undergoing mandatory military conscription examinations between 1969 and 2005 ( $n=1819$ 828), employing objective measurements of cardiovascular fitness at baseline.

- The study has a long follow-up time (range: 2-46 years) and relies on high-quality national register data to identify men, an understudied population in migraine research, who later develop prescription-requiring migraine.

- The incidence of migraine in our study is likely conservative since men with undiagnosed migraine or those only using over-the-counter or preventative migraine medication are not captured in the available data.

- Although analyses were adjusted for several important potential confounding variables, some residual confounding may remain in the estimates (such as smoking and alcohol consumption).

out prolonged strenuous exercise, has been shown to have beneficial effects on vascular health as well as associated with a reduced incidence of several neurological and cardiovascular disorders. ${ }^{2-4}$ However, data on the relationship between cardiovascular fitness and the development of migraine are lacking. Higher levels of physical activity, a behaviour in which body movement produced by muscle action increases the energy expenditure and enhances or maintains cardiovascular fitness, ${ }^{2}$ have been prospectively associated with a reduced risk of migraine. ${ }^{5}$ Physical activity is strongly correlated but is not interchangeable with cardiovascular fitness, as the latter also depends on other components such as genetic makeup. ${ }^{2}$

Although uninformative regarding direction of causality, cross-sectional studies have 
shown an inverse association of peak oxygen uptake with migraine,${ }^{6}$ whereas findings from cross-sectional studies of migraine and physical activity have been heterogeneous. ${ }^{7-10}$ Among individuals with migraine, regular physical activity appears to have alleviating effects on migraine symptoms such as frequency and intensity. ${ }^{11}$ However, acute physical exercise may also be a trigger of migraine episodes, ${ }^{12}$ and individuals with migraine or severe headaches might therefore be more reluctant to exercise. There is also a high comorbidity between migraine and cardiovascular conditions. ${ }^{13}$ While factors affecting cardiovascular health, such as weight and blood pressure, have also been shown to influence migraine prevalence, ${ }^{14} 15$ the contribution of cardiovascular fitness to long-term migraine risk may be differential across strata of body mass index (BMI) and blood pressure, though this has yet to be formally assessed.

Migraine is two to three times more prevalent in women, and findings on factors influencing migraine in men are scarce. ${ }^{16}$

The primary aim of this study was to estimate the effect of baseline cardiovascular fitness level on the longterm risk of prescription-requiring migraine in a large, prospective population-based cohort of young Swedish men with objective measures of cardiovascular fitness at baseline and a long follow-up time. The secondary aim was to evaluate whether the relationship between cardiovascular fitness and future risk of migraine is modified by baseline levels of BMI or blood pressure.

\section{METHODS}

We performed a population-based prospective study of young Swedish men enlisting for military service. Exposure variables were obtained at conscription (baseline) from records in the Swedish Military Service Conscription Register. Data from this register were linked to the Swedish Prescribed Drug Register, the National Hospital Registers, the Longitudinal Integration Database for Health Insurance and Labour Market Studies and the Swedish Cause of Death Register. Linkage of individual data was made possible by the unique personal identification number assigned to each registered person in Sweden. After linkage, all data were anonymised and coded by Statistics Sweden to maintain the confidentiality for the included men.

During follow-up, use of migraine-specific medication identified through the Swedish Prescribed Drug Register served as a proxy for migraine. Further, in a secondary analysis, hospital diagnoses of migraine, identified in the National Hospital Register, were recorded as outcomes.

\section{Study population}

The source population of the study comprised all men ( $\mathrm{n}=1 \quad 819$ 828) who enlisted for military service during 1968-2005, were 16-25 years old and had a documented test centre location. Enlistment was mandatory during this period for all Swedish men. Only individuals with severe

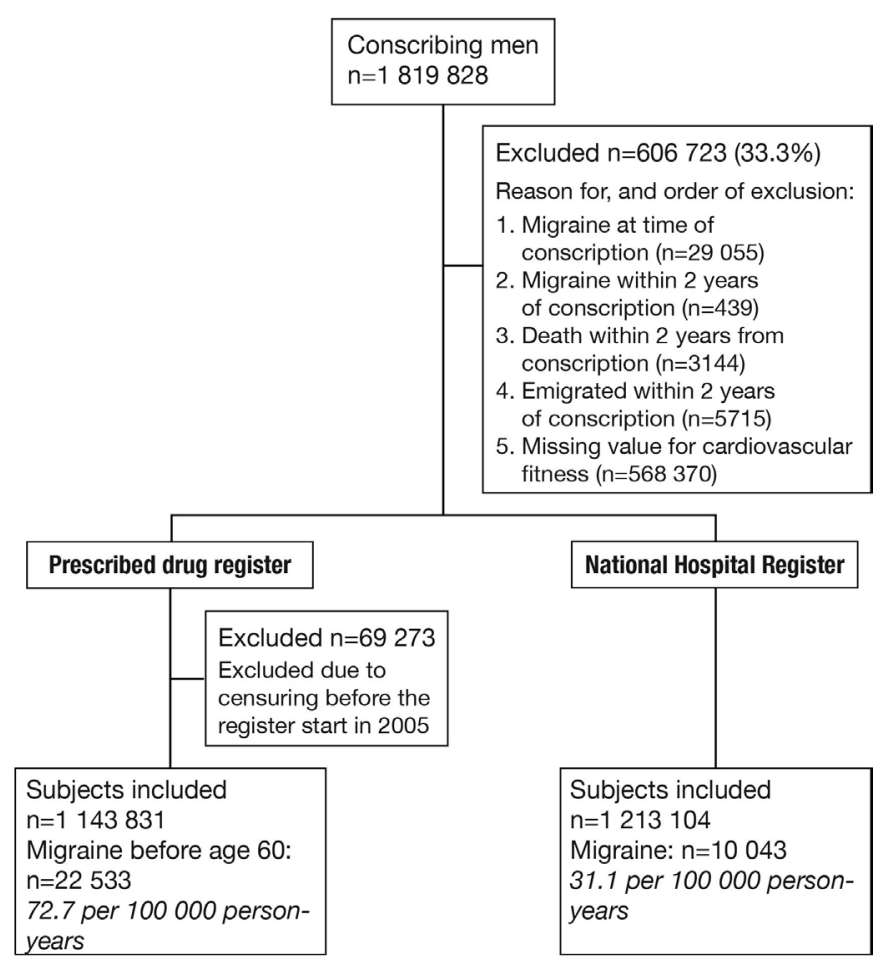

Figure 1 Flowchart of the study populations showing included and excluded subjects and number of outcomes.

chronic medical or mental conditions, serious disabilities or incarceration were granted exemption (in all, 2\%-3\% of the male population per year). The vast majority were 18 years old at time of conscription (mean age 18.2, $\mathrm{SD}=0.7$ ). To reduce the risk of possible reverse causation, men with a prescribed migraine-specific medication or migraine diagnosis prior to or during the 2 years after conscription (identified through the National Hospital Register and Conscription Register) were excluded from all analyses, as were men who died or emigrated within 2 years of conscription, as well as individuals with missing data on cardiovascular fitness (figure 1). For analyses using the Prescribed Drug Register, men who died or emigrated prior to the start of the register (2005) were excluded (figure 1).

\section{Ascertainment of exposure: cardiovascular fitness}

All men underwent a 2-day examination at one of six Swedish conscription centres (Southern, Western, Eastern, Central/Bergslagen, Northern lower and Northern upper). Cardiovascular fitness was evaluated at all centres for all included years. Cardiovascular fitness was objectively measured by a physician using a standardised maximal cycle ergometric test. Following 5 min of warm-up, the work rate was increased continuously by $25 \mathrm{~W} / \mathrm{min}$ until exhaustion. The final work rate (Wmax) was recorded, divided by body weight and then converted into a stanine score, which served as a measure of cardiovascular fitness. We have previously observed that the frequency distribution of cardiovascular fitness in the dataset is right skewed and not normally distributed. Therefore, as in other studies, ${ }^{17}$ cardiovascular 
fitness categories were trichotomised as low (score 1-4), medium (score 5-7) and high (score 8-9). Although the protocol for the ergometer test has changed over the years, average cardiovascular fitness scores have remained stable over time $(<1 \%$ change). The conscription cardiovascular fitness test has also been shown to have good reliability and validity. ${ }^{18}$

\section{Covariates}

Weight, height and systolic and diastolic blood pressures were measured by a physician following a written standard protocol. Systolic and diastolic blood pressures were measured on the first conscription day after $5-10$ min rest in the supine position. One measurement was generally performed, although when systolic blood pressure was over $145 \mathrm{~mm} \mathrm{Hg}$ or diastolic blood pressure was outside the range of $50-85 \mathrm{~mm} \mathrm{Hg}$, a second measurement was made on the following day and included in the register instead. Weight $(\mathrm{kg})$ and height $(\mathrm{m})$ were measured and BMI was calculated as $\left(\mathrm{kg} / \mathrm{m}^{2}\right)$. BMI values $<10$ and $>60 \mathrm{~km} / \mathrm{m}^{2}$ were treated as implausible values and excluded. BMI was categorised as low $\left(<18.5 \mathrm{~kg} / \mathrm{m}^{2}\right)$, normal $(18.5-24.9 \mathrm{~kg} /$ $\left.\mathrm{m}^{2}\right)$ and high $\left(>25.0 \mathrm{~kg} / \mathrm{m}^{2}\right)$. Systolic and diastolic blood pressures were divided into three groups; systolic blood pressure: $\geq 140,120-139$ and $\leq 119 \mathrm{~mm} \mathrm{Hg}$ and diastolic blood pressure: $\geq 80,66-79$ and $\leq 65 \mathrm{~mm} \mathrm{Hg}$. Information on conscription test centre, conscription year, age at conscription and parental education level were also taken into account as potential sources of confounding. We anticipated differences in conscription procedures and diagnosis frequencies depending on enlistment year. Socioeconomic status has also been shown to affect level of cardiovascular fitness in the current dataset and has been associated with migraine risk. ${ }^{19}$ Therefore, we controlled our analyses for parental education as a proxy for socioeconomic status. Information on parental education $(80 \%$ coverage), as well as emigration, were collected from the Longitudinal Integration Database for Health Insurance and Labour Market Studies (Swedish acronym LISA; http://www.scb.se/Pages/List__257743.aspx) at Statistics Sweden. The LISA database includes data from all Swedish residents aged 16 years and older and is annually updated. Parental education information (maternal and paternal education treated separately) was graded in three levels: prehigh school education (up to 9 years), high school education and university/postgraduate education.

\section{Outcome variables}

The outcome of migraine between age 20 and 60 was obtained using first recorded dispensing of prescribed migraine-specific medication, identified using the Swedish Prescribed Drug Register. This national register started in 2005 and includes detailed information on all prescription drugs (from primary care and hospital-based care) dispensed by all pharmacies in Sweden. ${ }^{20}$ The Prescribed Drug Register is updated monthly, and the prescriptions are grouped according to the Anatomical
Therapeutic Chemical classification (WHO). The following migraine-specific medications were included: sumatriptan (N02CC01), naratriptan (N02CC02), zolmitriptan (N02CC03), rizatriptan (N02CC04), almotriptan (N02CC05), eletriptan (N02CC06), frovatriptan (N02CC07), dihydroergotamine (N02CA01) and ergotamine (N02CA02). As these medications are also indicated for cluster headache, men with this diagnosis (International Classification of Diseases (ICD)-10: G44.0; ICD-9: 346C; ICD-8: 346.01) in the National Hospital Register were excluded from the analyses.

Secondary analyses were additionally performed using ICD codes for migraine diagnosis from the Swedish National Hospital Register as an outcome (ICD-10: G43; ICD-9: 346A,B,X,W; ICD-8: 346.09). This register contains both inpatient and outpatient diagnoses recorded in a hospital setting including referrals to neurologists/ migraine specialists and emergency visits. In Sweden, it is mandatory for all private and publicly funded hospitals to register one principal diagnosis at discharge and up to 30 contributory diagnoses. Register coverage for all inpatient care increased gradually during 1968-1986, and diagnoses from hospital outpatient care were documented starting in 2001. The Swedish National Hospital Register is a national, population-based register with high coverage; it is validated with positive predictive values of $(85 \%-95 \%)$ for most ICD diagnoses. ${ }^{21}$

\section{Statistical analyses}

To address the primary aim that is, how cardiovascular fitness at baseline affects risk of migraine during follow-up, we calculated risk ratios (RRs) and 95\% CIs using Poisson regressions, with exposure time as an offset to correct for differences in exposure time, and robust SEs. Using these models, we also calculated the maximum likelihood estimations for the adjusted population attributable fractions (PAF) with corresponding 95\% CIs. ${ }^{22} 23$ We here interpret PAFs as the estimated percentage of all cases of the outcome during follow-up that could have been prevented if the men of specified cardiovascular fitness group had instead belonged to the group with high cardiovascular fitness. We chose to perform RR analyses due to the aggregation of both new and older records in the Prescribed Drug Register at register initiation. For this reason, it was not possible to establish the exact time of first prescription, rendering Cox proportional hazards models unsuitable.

All analyses were adjusted for age at conscription, conscription region, conscription decade and parental education (model 1). Given that BMI might affect both cardiovascular fitness and migraine, we also performed an additional model (model 2) additionally adjusting for $\mathrm{BMI},{ }^{24}$ though there is uncertainty about the directionality of this relationship. To address our secondary aim that is, whether the relationship of cardiovascular fitness and migraine was modified by levels of BMI or blood pressure, we stratified the risk estimates from model 1 by categories of baseline BMI, as well as systolic and diastolic 
blood pressure, as previously defined. Missing data on parental education levels were treated as additional, separate category. High cardiovascular fitness was used as the reference category.

We also performed secondary analyses to evaluate the relationship between cardiovascular fitness and risk of hospital-based migraine diagnosis, which likely includes the most severe migraine cases. Since time of diagnosis information was available in this register, Cox proportional hazards models were used to estimate HRs and 95\% CIs. The follow-up period began at conscription (baseline), and person-time was included until time of (1) first record of migraine in the National Hospital Register, (2) death, (3) emigration or (4) at the end of follow-up, that is, on 31 December 2014, whichever happened first. Observed follow-up ranged from a minimum of 2 years to a maximum 46 years. All death dates were obtained from the Swedish Cause of Death Register, which has kept record of virtually all deaths in Sweden since 1961. We performed separate analyses for outpatient migraine diagnoses only, as well as outpatient and inpatient diagnoses, together. By removing the inpatient diagnoses, individuals with the highest degree of psychiatric and somatic comorbidities, medication overuse and highest pain scores were excluded ${ }^{25}$ rendering the patient group more homogeneous. We also performed a separate set of analyses including only primary diagnoses of migraine.

All analyses were conducted using SAS V.9.4 (SAS Institute).

\section{Patient and public involvement}

There was no patient involvement in this register-based study.

\section{RESULTS}

Baseline characteristics for the full study population and stratified by levels of cardiovascular fitness in the study population are shown in table 1 . Men with lower cardiovascular fitness levels were more likely to have lower parental education, higher BMI and higher systolic blood pressure than men with higher fitness. After we applied the exclusion criteria, analyses of dispensed migraine-specific medication were conducted using data from a total of 1143831 participants. Secondary analyses of migraine diagnoses documented in the National Hospital Register were conducted with 1213104 included participants (figure 1).

\section{Cardiovascular fitness and future dispensing of migraine- specific medication}

Prescriptions for migraine-specific medication were filled by 22533 of the included men (2.0\%). Compared with those with a high cardiovascular fitness level at conscription, both low and medium levels of fitness were associated with an increased risk for use of migraine-specific medication during follow-up (adjusted RR 1.29, 95\% CI 1.24 to 1.35 and $1.15,95 \%$ CI 1.12 to 1.19 ; table 2 ). The PAF of medium cardiovascular fitness for migraine-specific medication $(8.0 \%)$ was higher than that of low fitness $(3.6 \%)$. Including BMI as an additional potential source of confounding in the multivariable models did not change the RRs or PAFs substantially. The proportion of men with missing values for cardiovascular fitness was $31.6 \%$ for men with migraine-specific medication and $32.4 \%$ for men without.

\section{Risks stratified by categories of BMI and blood pressure}

The association of cardiovascular fitness with future migraine-specific medication was analysed in separate strata of BMI and blood pressure groups to assess potential effect measure modification (figure 2). Low and medium fitness were associated with an increased risk for migraine medication prescription among men with high and normal BMI, but no increased risk for the outcome was observed among underweight men. On stratification for systolic blood pressure levels, low and medium fitness had similar associations with future migraine medication across all three categories. Fitness was not associated with future migraine-specific medication prescription in men with high diastolic blood pressure $(\geq 80 \mathrm{~mm} \mathrm{Hg})$ but was associated with increased risk of prescription in men with lower diastolic blood pressures (in $66-79 \mathrm{~mm} \mathrm{Hg}$ as well as $\leq 65 \mathrm{~mm} \mathrm{Hg}$ groups).

\section{Cardiovascular fitness and future migraine diagnosis}

Migraine diagnoses were recorded in the National Hospital Register for 10043 men $(0.8 \%$ of the entire study population). Most (82\%) were primary diagnoses. Approximately one-third of the men with a migraine diagnosis in the National Hospital Register were also observed to have a migraine medication prescription in the Prescribed Drug Register. Lower cardiovascular fitness was found to increase the risk of a first-time migraine outpatient diagnosis (table 3 ). Adding migraine codes for inpatient migraine to the outpatient codes did not result in a substantial change in our findings. Associations were similar when analysing only primary migraine diagnoses. For primary outpatient diagnoses, the HR for low and medium cardiovascular fitness were 1.15 , 95\% CI 1.05 to 1.26 and $1.07,95 \%$ CI 1.01 to 1.14 , respectively. For combined primary outpatient and inpatient diagnoses, the HR for low fitness was $0.98,95 \%$ CI 0.91 to 1.09 and HR for medium fitness was 1.03 , $95 \%$ CI 0.98 to 1.09 .

\section{DISCUSSION}

In this large, prospective cohort of young adult men, cardiovascular fitness was inversely associated with future prescription-requiring migraine. Though quite similar, the risks were somewhat larger for filled migraine-specific drug prescriptions than for outpatient migraine diagnosis. The increased relative risk of migraine among those with a low level of cardiovascular fitness was higher than for those with a medium level fitness. However, more new migraine cases detected in this study were attributable to medium cardiovascular fitness, which was more common in the study population, rather than to low cardiovascular fitness. 
Table 1 Baseline characteristics of the study population, stratified by cardiovascular fitness level in a cohort of 1142831 male conscripts included in the analyses of migraine-specific medication prescription before age 60

\begin{tabular}{|c|c|c|c|c|}
\hline & & Level of cardio & ular fitness* & \\
\hline & All, $n(\%)$ & High, n (\%) & Medium, n (\%) & Low, n (\%) \\
\hline Total & $1143831(100.0)$ & $316113(100.0)$ & $673536(100.0)$ & $154182(100.0)$ \\
\hline Migraine-specific medication & $22533(2.0)$ & $5525(1.7)$ & $13448(2.0)$ & $3560(2.3)$ \\
\hline Decade of conscription & & & & \\
\hline 1968-1969 & $9619(0.8)$ & $2920(0.9)$ & $5169(0.8)$ & $1530(1.0)$ \\
\hline 1970 s & $357123(32.8)$ & 123367 (39.0) & 198357 (29.5) & 53399 (34.6) \\
\hline 1980s & $432857(37.8)$ & $104672(33.1)$ & $252082(37.4)$ & $76103(49.4)$ \\
\hline $1990 s$ & $247079(21.6)$ & 65057 (20.6) & $161868(24.0)$ & 20154 (13.1) \\
\hline $2000 s$ & $79153(6.9)$ & $20097(6.4)$ & $56060(8.3)$ & $2996(1.9)$ \\
\hline Place of conscription & & & & \\
\hline South & 263865 (23.1) & $70546(22.3)$ & $158784(23.6)$ & 34535 (22.4) \\
\hline West & 245545 (21.5) & $62247(19.7)$ & $145540(21.6)$ & $37758(24.5)$ \\
\hline East & $246449(21.5)$ & $68731(21.7)$ & $140660(20.9)$ & $37058(24.0)$ \\
\hline Bergslagen & $225046(19.7)$ & $63472(20.1)$ & 130994 (19.4) & 30580 (19.8) \\
\hline Lower Norrland & $105809(9.3)$ & $31230(9.9)$ & $64667(9.6)$ & $9912(6.4)$ \\
\hline Upper Norrland & $57117(5.0)$ & $19887(6.3)$ & $32891(4.9)$ & $4339(2.8)$ \\
\hline Parental education & & & & \\
\hline Prehigh school & $345478(30.2)$ & $83976(26.6)$ & $199592(29.6)$ & $61910(40.2)$ \\
\hline High school & $478024(41.8)$ & $124718(39.5)$ & $289883(43.0)$ & 63424 (41.1) \\
\hline University or higher & $290792(25.4)$ & $100633(31.8)$ & $166772(24.8)$ & $23388(15.2)$ \\
\hline Missing & $29537(2.6)$ & $6788(2.1)$ & $17289(2.6)$ & $5460(3.5)$ \\
\hline BMI & & & & \\
\hline High $(\geq 25.0)$ & $89073(7.8)$ & $6344(2.0)$ & $52873(7.9)$ & $29856(19.4)$ \\
\hline Normal (18.5-24.9) & 930230 (81.3) & 283599 (89.7) & 549055 (81.5) & 97578 (63.3) \\
\hline Low $(<18.5)$ & $121670(10.6)$ & $25703(8.1)$ & $69896(10.4)$ & 26071 (16.9) \\
\hline Missing & $2858(0.2)$ & $469(0.1)$ & $1712(0.3)$ & $677(0.4)$ \\
\hline Systolic blood pressure & & & & \\
\hline$\geq 140 \mathrm{~mm} \mathrm{Hg}$ & $211550(18.5)$ & $50693(16.0)$ & $128388(19.1)$ & $32469(21.1)$ \\
\hline $120-139 \mathrm{~mm} \mathrm{Hg}$ & $720456(63.0)$ & 199746 (63.2) & $424041(63.0)$ & $96678(62.7)$ \\
\hline$\leq 119 \mathrm{~mm} \mathrm{Hg}$ & $206286(18.0)$ & $64170(20.3)$ & 117757 (17.5) & $24360(15.8)$ \\
\hline Missing & $5530(0.5)$ & $1505(0.5)$ & $3350(0.5)$ & $675(0.4)$ \\
\hline Diastolic blood pressure & & & & \\
\hline$\geq 80 \mathrm{~mm} \mathrm{Hg}$ & $476880(41.7)$ & $130450(41.3)$ & $283226(42.1)$ & $63204(41.0)$ \\
\hline $66-79 \mathrm{~mm} \mathrm{Hg}$ & $506599(44.3)$ & $140125(44.3)$ & $297911(44.2)$ & $68564(44.5)$ \\
\hline$\leq 65 \mathrm{~mm} \mathrm{Hg}$ & $154631(13.5)$ & 43959 (13.9) & $88962(13.2)$ & $21710(14.1)$ \\
\hline Missing & $5721(0.5)$ & $1580(0.5)$ & $3437(0.5)$ & $704(0.5)$ \\
\hline
\end{tabular}

*Performance was trichotomised as low (score 1-4), medium (score 5-7) and high (score 8-9).

BMI, body mass index.

\section{Comparisons with other studies}

This longitudinal, population-based study provides new insights into the effects of cardiovascular fitness on migraine risk. While not directly comparable with cardiovascular fitness, two prospective studies have previously evaluated the relationships between physical activity and migraine with inconsistent results. ${ }^{58} \mathrm{We}$ emphasise the importance of distinguishing cardiovascular fitness from physical activity. Cardiovascular fitness is more strongly related to health outcomes than physical activity, and activity not resulting in an increase in fitness level may not provide protective effects against adverse health outcomes. ${ }^{26}$ For this reason, studies of cardiovascular fitness in addition to physical activity are of great 
Table 2 Risk ratios and population attributable fractions for prescribed migraine-specific medication before the age of 60 in relation to cardiovascular fitness levels in young adult men

\begin{tabular}{|c|c|c|c|}
\hline & Model $1^{*}, \mathrm{n}=1143831$ & & \\
\hline Cardiovascular fitness & Migraine /total no. & RR $(95 \% \mathrm{Cl})$ & PAF $(95 \% \mathrm{Cl})$ \\
\hline High & $5525 / 316113$ & 1.00 (reference) & \\
\hline Medium & $13448 / 673536$ & 1.15 (1.12 to 1.19$)$ & $8.0 \%(4.0$ to 11.7$)$ \\
\hline \multirow[t]{2}{*}{ Low } & $3550 / 154182$ & 1.29 (1.24 to 1.35$)$ & $3.6 \%$ (1.7 to 5.3$)$ \\
\hline & \multicolumn{2}{|c|}{ Model 2‡, n=1 140973} & \\
\hline Cardiovascular fitness $†$ & Migraine /total no. & RR $(95 \% \mathrm{Cl})$ & PAF $(95 \% \mathrm{Cl})$ \\
\hline High & $5517 / 315644$ & 1.00 (reference) & \\
\hline Medium & $13411 / 671824$ & 1.14 (1.11 to 1.18$)$ & $7.5 \%$ (3.2 to 11.4$)$ \\
\hline Low & $3537 / 153505$ & 1.27 (1.21 to 1.32$)$ & $3.3 \%$ (1.2 to 5.2$)$ \\
\hline
\end{tabular}

${ }^{*}$ Adjusted for age, conscription calendar year and region and parental education.

†High level=reference category.

¥Adjusted for age, conscription calendar year and region, parental education and BMI.

.BMI, body mass index; PAF, population attributable fraction; RR, risk ratio; no., number of events.

importance. A cross-sectional study found an inverse relationship of peak oxygen uptake and migraine in adults younger than $50,{ }^{6}$ but given its cross-sectional study

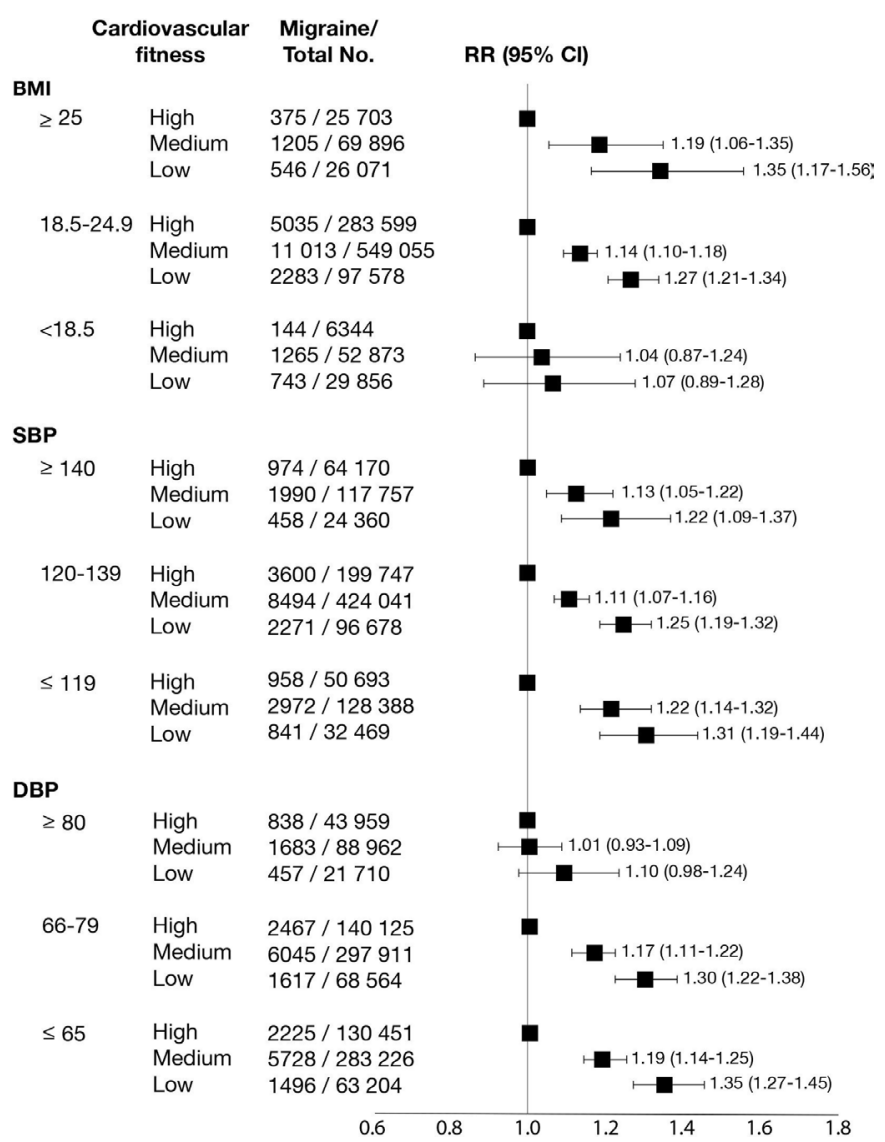

Figure 2 RRs of prescribed migraine-specific medication in relation to cardiovascular fitness levels stratified by categories of BMI, SBP and DBP at baseline. RR adjusted for age, conscription calendar year and region parental education. BMI, body mass index; DBP, diastolic blood pressure; no., number of events; RR, risk ratio; SBP, systolic blood pressure. design, no conclusions can be made regarding the direction of the observed effect.

\section{Effect measure modification by BMI and blood pressure}

The association of cardiovascular fitness and migraine was only observed in men with normal and high BMI but not in those with low BMI. Prospective studies of BMI and later risk of migraine appear to be lacking, but a meta-analysis of cross-sectional studies concluded that the risk of migraine appears to be moderately increased in both obese and underweight individuals. ${ }^{14}$ Our results suggest that among men with a low BMI at age 18, there was no additional beneficial effect of having a higher cardiovascular fitness with respect to prescription-requiring migraine risk later in life. It should, however,

Table 3 HRs of migraine diagnoses, recorded in the National Hospital register, in relation to cardiovascular fitness levels in young adult men followed for up to 46 years

\begin{tabular}{lll}
\hline \multicolumn{2}{l}{ Outpatient migraine diagnoses only } & \\
\hline $\begin{array}{l}\text { Cardiovascular } \\
\text { fitness* }\end{array}$ & $\begin{array}{l}\text { Migraine/total } \\
\text { no. }\end{array}$ & HR (95\% Cl) $\dagger$ \\
\hline High & $1759 / 338295$ & $1.00($ reference $)$ \\
Medium & $4060 / 710865$ & $1.08(1.02$ to 1.15$)$ \\
\hline Low & $1044 / 163946$ & $1.18(1.08$ to 1.28$)$ \\
\hline
\end{tabular}

All migraine diagnoses (inpatient and outpatient)

\begin{tabular}{lll}
\hline $\begin{array}{l}\text { Cardiovascular } \\
\text { fitness }\end{array}$ & $\begin{array}{l}\text { Migraine/total } \\
\text { no. }\end{array}$ & HR (95\% Cl) $\boldsymbol{~}$ \\
\hline High & $2655 / 338295$ & 1.00 (reference) \\
Medium & $5852 / 710865$ & $1.05(1.00$ to 1.12$)$ \\
Low & $1536 / 163946$ & $1.05(0.98$ to 1.12$)$ \\
\hline
\end{tabular}

*High level=reference category.

†Adjusted for age, conscription calendar year and region and parental education.

.BMI, body mass index;no., number of events. 
be noted that the number of men in this category was limited. It is also possible that there might be a common underlying factor influencing both BMI and migraine.

While an association between lower cardiovascular fitness and later migraine was observed across all categories of systolic blood pressure, this was not the case for the group with a diastolic blood pressure of $\geq 80 \mathrm{~mm} \mathrm{Hg}$. This observation may partly be explained by the previously reported relationship between higher blood pressures and hypoalgesia. ${ }^{27}$ It could also reflect an unknown, underlying cause of migraine in young men with extreme blood pressures. Additional studies are needed to further elucidate the modifying effects of BMI and blood pressure on the inter-relationship between cardiovascular fitness and migraine.

\section{Possible mechanisms}

The increased risk of migraine among men with lower cardiovascular fitness at age 18 observed in this study may be explained by a combination of several factors. There might be an unknown, common predisposing factor for both lower cardiovascular fitness levels and migraine given the known, clear association of migraine with unfavourable cardiovascular risk factor profiles. ${ }^{28}$ An association between migraine and cardiovascular risk comorbidities could be explained by a common pathology underlying both conditions, and migraine might be a local manifestation of a systemic, rather than neurological, phenomenon. ${ }^{28}$ Our research findings expand on previous studies linking migraine to cardiovascular risk factor profiles, highlighting the long-term association between cardiovascular fitness and migraine.

Several areas of the brain are still developing during late adolescence, and it is also possible that cardiovascular fitness during this period has long-term effects on brain health that might reduce susceptibility to migraine. Low cardiovascular fitness has been shown to increase the risk of several other neurological and psychiatric disorders such as stroke, ${ }^{3}$ epilepsy ${ }^{4}$ and depression. ${ }^{29}$ It could be that a common mechanism affecting neuroprotection, neurogenesis, synaptic plasticity, neuroinflammation and neurotrophic factors such as brain-derived neurotrophic factor (BDNF) may be involved. ${ }^{30}$ Hence, higher cardiovascular fitness might result in a greater 'brain reserve' that may act as a compensatory buffer of brain plasticity and neural resources and better enable the brain to cope with neuropathology, resulting in long-lasting beneficial effects on brain health. ${ }^{31}$ Indeed, there is increasing evidence suggesting that behavioural interventions such as physical activity during critical stages of development can have such long-lasting and robust effects on the brain. ${ }^{31}$

Cardiovascular fitness may influence specific aspects of migraine burden contributing to severity. Several studies report lower migraine burden with more physical activity, ${ }^{32}$ and a recent randomised study of exercise in migraine patients showed decreased migraine pain, intensity and frequency after the training intervention. ${ }^{33}$
Neuroadaptive changes with pain are well-known, ${ }^{34}$ and decreased levels of BDNF have been reported in patients with migraine. ${ }^{35}$ Higher fitness levels during adolescence could contribute to long-lasting structural and/or biochemical changes to the brain, generating a higher threshold for migraine pain.

\section{Strengths and limitations of this study}

Strengths of this study include the population-based design, its large size and the objective measurements of cardiovascular fitness at baseline. Other strengths are available information on a large number of important covariates, the long available follow-up time and the linkage of national register data to identify conscripts who later developed migraine. Furthermore, our study focuses on men, an understudied population in migraine research.

Several limitations should, however, be considered when interpreting our results. We show that $2 \%$ of the men filled a first-time prescription of migraine-specific medication during follow-up. However, the mean prevalence of current migraine among adult men in Europe has been estimated to be $8 \%$ in a review combining studies with variable timeframes. ${ }^{36}$ Although our figures show first-time prescriptions, these should not be interpreted as equivalent to prevalence. Since our outcome measurements only capture men who seek healthcare and we are unable to identify men using over-thecounter migraine medication only, no medication at all, or preventative medications, incidence of migraine in the current study is likely underestimated. The included men classified as having the outcome likely have more severe migraine episodes, which cannot be managed by the patient himself.

Although we excluded men who died or emigrated before the Prescribed Drug Register started in 2005, we may have missed men who enlisted during the earlier years and had migraine that later resolved (or men who did not seek treatment for migraine later). Boys with an early migraine diagnosis that resolved prior to conscription might have been misclassified as having migraine at baseline and would hence be wrongfully excluded from the analyses. Although we have attempted to reduce the risk of possible reverse causation by excluding men with migraine prior to or during the 2 years after conscription, there is still a possibility that adolescent men had migraine but were not diagnosed and had not received prescribed migraine medication. Such men have been included in the current study, and this could influence cardiovascular fitness if they were less active due to their migraine.

The hospital register does not include codes from primary care; therefore, men suffering from migraine without hospital-based care could only be otherwise identified through the Prescribed Drug Register. In Sweden, migraine or cluster headache are the only indications for triptans and ergotamide (Farmaceutiska Specialiteter i Sverige (FASS); https://www.fass.se/). 
We have excluded men with prescribed migraine-specific medication at baseline as well as men diagnosed with cluster headache. However, there is still a small possibility that these medications could be prescribed for other indications, and these participants could be misclassified as having the outcome of interest in our analyses using the Prescribed Drug Register data. The migraine diagnosis based on ICD codes has not been formally validated in the Swedish Hospital Register. However, we have no reason to believe that the generally high positive predictive values observed for other ICD codes in this register would not also apply to migraine.

Although we have controlled for confounding by adjusting for information on several covariates, residual and unmeasurable confounding may still be present. In particular, we had no information on smoking and alcohol consumption.

Our research goal was to estimate the effect of baseline cardiovascular fitness on long-term risk of migraine. Therefore, we do not include data on cardiovascular fitness or other health-related risk factors at later stages in life. We have no information regarding changes in this variable over time. An additional limitation is that, due to the identification method of migraine used, we were not able to stratify subjects according to migraine frequency or severity, episode duration or aura status. Hence, we could not assess how cardiovascular fitness influences the risk of different subtypes of migraine. We emphasise that our findings should not be interpreted as explanatory regarding the causal chain leading to the onset of migraine.

We acknowledge the relatively large number of missing values for cardiovascular fitness exposure variable. However, since proportions with missing values were very similar among men with and without migraine-specific medication, we think it is unlikely this missingness would be differential. Since the main purpose of the Swedish Conscription Register was for military use, detailed descriptions of causes for missing data are classified, and we thus unfortunately cannot explore these reasons further. Lastly, we emphasise that since women have a different body composition, physiology, and clinical features of migraine,${ }^{16}$ our findings should not be directly extrapolated to women.

\section{CONCLUSION}

Lower cardiovascular fitness in adult young men was found to be associated with increased risk of future prescription-requiring migraine. Our study calls for future targeted research to assess whether interventions to improve cardiovascular fitness result in a reduced risk of developing migraine among men with low cardiovascular fitness levels. In addition, studies that explore possible mediators of the effect of cardiovascular fitness on the later development of migraine may provide important insights into the biological understanding of migraine, ultimately informing the development of further preventive strategies.

\section{Author affiliations}

${ }^{1}$ Center for Brain Repair and Rehabilitation, Institute of Neuroscience and Physiology, Sahlgrenska Academy, University of Gothenburg, Gothenburg, Sweden ${ }^{2}$ Region Västra Götaland, Sahlgrenska University Hospital, Neurology Clinic, Gothenburg, Sweden

${ }^{3}$ Department of Forensic Genetics and Forensic Toxicology, National Board of Forensic Medicine, Linköping, Sweden

${ }^{4}$ Department of Neuromedicine and Movement Science, NTNU Norwegian University of Science and Technology; Norwegian Advisory Unit on Headache, St Olavs University Hospital, Trondheim, Norway

${ }^{5}$ Department of Internal Medicine, Institute of Medicine, Sahlgrenska Academy, University of Gothenburg, Gothenburg, Sweden

${ }^{6}$ Region Västra Götaland, Sahlgrenska University Hospital, Department of Internal Medicine, Gothenburg, Sweden

${ }^{7}$ Institute of Public Health, Charité-Universitätsmedizin Berlin, Berlin, Germany ${ }^{8}$ Department of Primary Health Care, Institute of Medicine, Sahlgrenska Academy, University of Gothenburg, Gothenburg, Sweden

${ }^{9}$ Region Västra Götaland, Närhälsan, Gothenburg, Sweden

${ }^{10}$ Department of Psychiatry and Neurochemistry, Institute of Neuroscience and Physiology, Sahlgrenska Academy, University of Gothenburg, Gothenburg, Sweden

${ }^{11}$ Region Västra Götaland, Sahlgrenska University Hospital, Psychosis Clinic, Gothenburg, Sweden

${ }^{12}$ Center for Stroke Research and Neurocure Cluster of Excellence, Charité Universitätsmedizin Berlin, Berlin, Germany

Contributors JN, SG, ML, NDÅ, MÅ, MW and GHK conceptualised the study. JN, SG, NDÅ, MÅ, MW and GHK contributed to data acquisition. JN, SG, JLR and TK designed the analyses, and JN and SG performed the analyses. All coauthors contributed to interpretation of as well as drafting and critically revising the manuscript for important intellectual content. JN, SG and GHK are the guarantors.

Funding This work was supported by the Swedish state under the agreement between the Swedish government and the county councils, the ALF agreement (ALFGBG-726541, ALFGBG-715841), the Swedish Brain Research Foundation (Hjärnfonden), the Swedish Research Council (521-2014-3224) and the Stiftelsen Peter Erikssons minnesfond för hjärnforskning. JR's research position is funded by a grant from the Else-Kröner-Fresenius Stiftung (GSO/EKFS-17, granted to TK).

Competing interests TK reports having contributed to an advisory board of CoLucid and a research project funded by Amgen, for which the Charité Universitätsmedizin Berlin received an unrestricted compensation. He further reports having received honoraria from Lilly, Newsenselab, and Total for providing methodological advice, from Novartis and from Daiichi Sankyo for providing a lecture on neuroepidemiology and research methods, and from the BMJ for editorial services. The other authors have no conflicts of interest.

Patient consent for publication Not required.

Ethics approval The Ethics Committee of the University of Gothenburg and Confidentiality Clearance at Statistics Sweden approved this study (Dnr 462-14).

Provenance and peer review Not commissioned; externally peer reviewed.

Data availability statement No data are available. All data relevant to the study are included in the article or uploaded as supplementary information.

Open access This is an open access article distributed in accordance with the Creative Commons Attribution Non Commercial (CC BY-NC 4.0) license, which permits others to distribute, remix, adapt, build upon this work non-commercially, and license their derivative works on different terms, provided the original work is properly cited, appropriate credit is given, any changes made indicated, and the use is non-commercial. See: http://creativecommons.org/licenses/by-nc/4.0/.

\section{REFERENCES}

1. Jacobs B, Dussor G. Neurovascular contributions to migraine: moving beyond vasodilation. Neuroscience 2016;338:130-44.

2. Ortega FB, Ruiz JR, Castillo MJ, et al. Physical fitness in childhood and adolescence: a powerful marker of health. Int $J$ Obes 2008;32:1-11. 
3. Åberg ND, Kuhn HG, Nyberg J, et al. Influence of cardiovascular fitness and muscle strength in early adulthood on long-term risk of stroke in Swedish men. Stroke 2015;46:1769-76.

4. Nyberg J, Aberg MAI, Toren K, et al. Cardiovascular fitness and later risk of epilepsy: a Swedish population-based cohort study. Neurology 2013;81:1051-7.

5. Hagen K, Åsberg AN, Stovner L, et al. Lifestyle factors and risk of migraine and tension-type headache. Follow-up data from the NordTrøndelag health surveys 1995-1997 and 2006-2008. Cephalalgia 2018;38:1919-26.

6. Hagen K, Wisløff U, Ellingsen Øyvind, et al. Headache and peak oxygen uptake: the HUNT3 study. Cephalalgia 2016;36:437-44.

7. Lebedeva ER, Kobzeva NR, Gilev DV, et al. Factors associated with primary headache according to diagnosis, sex, and social group. Headache 2016;56:341-56.

8. Varkey E, Hagen K, Zwart J-A, et al. Physical activity and headache: results from the Nord-Trøndelag health study (HUNT). Cephalalgia 2008;28:1292-7.

9. Molarius A, Tegelberg A, Öhrvik J. Socio-economic factors, lifestyle, and headache disorders - a population-based study in Sweden. Headache 2008;48:1426-37.

10. Winter AC, Hoffmann W, Meisinger C, et al. Association between lifestyle factors and headache. J Headache Pain 2011;12:147-55

11. Baillie LE, Gabriele JM, Penzien DB. A systematic review of behavioral headache interventions with an aerobic exercise component. Headache 2014;54:40-53.

12. Kelman $L$. The triggers or precipitants of the acute migraine attack. Cephalalgia 2007;27:394-402.

13. Merikangas KR. Contributions of epidemiology to our understanding of migraine. Headache 2013;53:230-46.

14. Gelaye B, Sacco S, Brown WJ, et al. Body composition status and the risk of migraine: a meta-analysis. Neurology 2017;88:1795-804.

15. Tronvik E, Stovner LJ, Hagen $\mathrm{K}$, et al. High pulse pressure protects against headache: prospective and cross-sectional data (HUNT study). Neurology 2008;70:1329-36.

16. Vetvik KG, MacGregor EA. Sex differences in the epidemiology, clinical features, and pathophysiology of migraine. Lancet Neurol 2017;16:76-87.

17. Aberg MA, Nyberg J, Toren $\mathrm{K}$, et al. Cardiovascular fitness in early adulthood and future suicidal behaviour in men followed for up to 42 years.. Psychol Med 2013:1-10.

18. Nordesjö LO, Schéle R. Validity of an ergometer cycle test and measures of isometric muscle strength when prediction some aspects of military performance. Swedish Journal of Defence Medicine 1974;10.

19. Stewart WF, Roy J, Lipton RB. Migraine prevalence, socioeconomic status, and social causation. Neurology 2013;81:948-55.

20. Wettermark B, Hammar N, Fored CM, et al. The new Swedish Prescribed Drug Register--opportunities for pharmacoepidemiological research and experience from the first six months. Pharmacoepidemiol Drug Saf 2007:16:726-35.

21. Ludvigsson JF, Andersson E, Ekbom A, et al. External review and validation of the Swedish national inpatient register. BMC Public Health 2011:11:450.

22. Rückinger $\mathrm{S}$, von Kries $\mathrm{R}$, Toschke $\mathrm{AM}$. An illustration of and programs estimating attributable fractions in large scale surveys considering multiple risk factors. BMC Med Res Methodol 2009;9:7.

23. Greenland S, Drescher K. Maximum likelihood estimation of the attributable fraction from logistic models. Biometrics 1993;49:865-72.

24. Ornello R, Ripa P, Pistoia F, et al. Migraine and body mass index categories: a systematic review and meta-analysis of observational studies. J Headache Pain 2015;16:27.

25. Marmura MJ, Goldberg SW. Inpatient management of migraine. Curr Neurol Neurosci Rep 2015;15:13.

26. Lee D-C, Sui X, Ortega FB, et al. Comparisons of leisure-time physical activity and cardiorespiratory fitness as predictors of all-cause mortality in men and women. Br J Sports Med 2011;45:504-10.

27. Bruehl S, Chung OY. Interactions between the cardiovascular and pain regulatory systems: an updated review of mechanisms and possible alterations in chronic pain. Neurosci Biobehav Rev 2004;28:395-414.

28. Hamed SA. The vascular risk associations with migraine: relation to migraine susceptibility and progression. Atherosclerosis 2009;205:15-22.

29. Åberg MAI, Waern M, Nyberg $\mathrm{J}$, et al. Cardiovascular fitness in males at age 18 and risk of serious depression in adulthood: Swedish prospective population-based study. Br J Psychiatry 2012;201:352-9.

30. Voss MW, Vivar C, Kramer AF, et al. Bridging animal and human models of exercise-induced brain plasticity. Trends Cogn Sci 2013;17:525-44.

31. Nithianantharajah J, Hannan AJ. The neurobiology of brain and cognitive reserve: mental and physical activity as modulators of brain disorders. Prog Neurobiol 2009;89:369-82.

32. Irby MB, Bond DS, Lipton RB, et al. Aerobic exercise for reducing migraine burden: mechanisms, markers, and models of change processes. Headache 2016;56:357-69.

33. Krøll LS, Hammarlund CS, Linde M, et al. The effects of aerobic exercise for persons with migraine and co-existing tension-type headache and neck pain. A randomized, controlled, clinical trial. Cephalalgia 2018;38:1805-16.

34. Apkarian AV. The brain in chronic pain: clinical implications. Pain Manag 2011;1:577-86.

35. Blandini F, Rinaldi L, Tassorelli $C$, et al. Peripheral levels of BDNF and NGF in primary headaches. Cephalalgia 2006;26:136-42.

36. Stovner LJ, Andree C. Prevalence of headache in Europe: a review for the Eurolight project. J Headache Pain 2010:11:289-99. 